ORIGINAL ARTICLE

\title{
Histological examination has a major impact on macroscopic necropsy diagnoses
}

\author{
F D C Bernardi, P H N Saldiva, T Mauad
}

J Clin Pathol 2005;58:1261-1264. doi: 10.1136/jcp.2005.027953

See end of article for authors' affiliations

.....................

Correspondence to: Dr T Mauad, Department of Pathology, São Paulo University Medical School, Avenida Dr Arnaldo, 455 1 st Floor, Sao Paulo SP, 01246-903 Brazil; tmauad@usp.br

Accepted for publication 5 April 2005
Background: Necropsy is the gold standard for clinicopathological discrepancy studies and epidemiological surveys. Inadequate sampling or lack of tissue may hamper the final interpretation and quality of the necropsy.

Aim: To compare the histological and gross necropsy diagnoses of different organs.

Methods: A retrospective comparison of the provisional reports (gross findings only) and the final reports (after histological examination) of the necropsies performed at the department of pathology of the Hospital das Clínicas, Sao Paulo University, Brazil, a large tertiary care complex, in 2001. The total number of diagnoses listed for the lungs, heart, liver, pancreas, kidneys, and spleen were calculated. Findings were categorised into concordant/refined diagnosis, discordant/additional diagnosis, histology needed, and inconclusive.

Results: Three hundred and seventy one postmortem reports were analysed. There were 214 men and 157 women, with a mean age of 50.3 years, ranging from 1 to 92 . The lung received the highest number (954) and the pancreas the lowest number (390) of diagnoses. The highest frequencies of discrepancies between the gross and microscopic findings were found in the lung and the liver: $38.7 \%$ and $35.1 \%$, respectively. The brain had the lowest frequency of discrepancies. In a small number of cases, the final diagnosis could only be achieved through microscopic analysis, with the highest frequency being found in the kidneys (8.5\%).

Conclusions: Histological analysis has a major impact on previously performed gross diagnosis at necropsy, especially in the lungs, liver, and kidneys. Adequate sampling and histological analysis are important for necropsy quality.
$\mathrm{T}$ he importance of the necropsy as a tool for medical education and quality control is well established. ${ }^{1-3}$ The necropsy is the standard method of determining the cause of death when investigating clinicopathological discrepancies and the epidemiology of disease. ${ }^{45}$ However, pathologists are also aware that necropsy quality may greatly influence the final correct diagnosis, by hampering the adequate interpretation of findings. There is little research addressing this subject. ${ }^{6}$ The available studies show that necropsy quality may vary greatly, both in perinatal and adult necropsies. ${ }^{7-9}$

\section{"There is some debate as to whether or not the necropsy is complete without systematic histological sampling"}

An important issue related to necropsy quality is histological sampling. There is some debate as to whether or not the necropsy is complete without systematic histological sampling. Lack of sampling can occur because of cost limitations, ${ }^{10}$ lack of time or interest to deal with postmortem histology, increased turnaround times, or even in reaction to the media or the general public. ${ }^{11}$ There is little information about how lack of sampling can affect necropsy quality. For certain organs, such as the lung, it has been shown that there are considerable intraobserver and interobserver discrepancies in the diagnosis of bronchopneumonia using the naked eye and histology. ${ }^{12}$ Not taking these discrepancies into account, or lack of necropsy quality control can seriously limit the use of the necropsy as the "final audit". To our knowledge, there are few necropsy studies comparing gross and histological diagnoses in different organs. ${ }^{12-14}$

To address the issue of diagnostic changes between initial gross diagnosis and subsequent histological analysis in several organs, we reviewed the provisional and final reports of necropsies performed at our institution in 2001. Such a study could contribute to the debate on whether necropsy histology is necessary, in which organs it is most necessary, and whether it can provide diagnostic advances.

\section{METHODS}

Our study was performed at the department of pathology of the University of Sao Paulo, Brazil, which is linked to Hospital das Clínicas, a large volume high complexity centre, with an average annual necropsy rate of $60 \%$ in the past five years. ${ }^{15}$ In 2001, 1273 medical necropsies were performed, representing a hospital necropsy rate of $51.8 \%$. For our study, we retrospectively analysed and compared the provisional (gross findings only) and the final (after histological analysis) reports of the necropsies performed by the resident staff. The senior staff supervised the performance of all necropsies and assisted with the histological analysis and final reports. All the necropsies had a broad, although not systematic, sampling. Intrauterine deaths, perinatal, and malformation necropsies were excluded.

We obtained the following data for each patient: age, sex, major underlying disease, and immediate cause of death. For the comparative analysis between provisional and final reports, the following organs were considered: lung, heart, liver, kidney, spleen, pancreas, and brain. We counted the number of different diagnoses that each organ received at the necropsy. For each organ, the findings were categorised as:

(1) Concordant diagnosis.

(a) There was total concordance between the macroscopic and microscopic diagnoses; both diseased and normal states were considered. When a given organ was not mentioned, both in the provisional or final report, it was considered as presenting without significant changes in both analyses. 
Table 1 Total number of diagnoses listed for each organ and the frequencies (\% of total diagnoses) of the four major categories analysed

\begin{tabular}{llllll}
\hline Organ & $\begin{array}{l}\text { Total number of } \\
\text { diagnoses }\end{array}$ & $\begin{array}{l}\text { Discordant diagnoses } \\
\mathbf{N}(\%)\end{array}$ & $\begin{array}{l}\text { Concordant diagnoses } \\
\mathbf{N}(\%)\end{array}$ & $\begin{array}{l}\text { Histology needed } \\
\mathbf{N}(\%)\end{array}$ & $\begin{array}{l}\text { Remained inconclusive } \\
\mathbf{N}(\%)\end{array}$ \\
\hline Lung & 954 & $369(38.7)$ & $564(59.1)$ & $20(2.1)$ & $1(0.1)$ \\
Kidney & 597 & $181(30.3)$ & $364(61)$ & $51(8.5)$ & $1(0.2)$ \\
Liver & 573 & $201(35.1)$ & $341(59.5)$ & $29(5.2)$ & $1(0.2)$ \\
Heart & 570 & $140(24.6)$ & $401(70.3)$ & $9(2.0)$ & 0 \\
Spleen & 444 & $127(28.6)$ & $306(68.9)$ & $8(1.8)$ & $4(0.5)$ \\
Brain & 437 & $61(14)$ & $364(83.3)$ & $8(2.0)$ & $0.9)$ \\
Pancreas & 390 & $97(24.9)$ & $285(73.1)$ & & 0 \\
\hline
\end{tabular}

(b) The histological diagnosis was concordant and refined the gross diagnosis. For example: gross diagnosis, bronchopneumonia; histological diagnosis, aspiration pneumonia; or gross diagnosis, endocarditis; histological diagnosis, endocarditis as a result of tuberculosis infection.

(2) Discordant/additional diagnosis.

(a) Discordance between gross and histological analysis For example: gross diagnosis, bronchopneumonia; histological diagnosis, lung oedema; gross diagnosis, hepatic siderosis; histological diagnosis, steatosis.

(b) A microscopic alteration described in the final report that theoretically could have been seen at gross examination. For example: lung emphysema.

(c) The alteration described in the gross report was not found in the final report. For example: gross diagnosis, hepatomegaly as a result of leukaemic infiltration; histological diagnosis, no diagnosis listed.

(3) The diagnosis listed in the final report could only be performed after histological analysis. For example: in the pancreas, Langerhan's island hyperplasia; in the kidney, mesangioproliferative glomerulonephritis.

(4) Both gross provisional and final histology reports remained inconclusive regarding the diagnosis of a given organ.

We further analysed whether the major disease and the immediate cause of death listed in the provisional report were altered after microscopic examination. Findings were grouped as concordant plus diagnostic refinements, discordant/additional diagnosis, histological analysis needed for definition, and inconclusive.

\section{Statistical analysis}

Values are expressed as mean (ranges) and percentages. The frequencies of discordant versus concordant diagnoses were compared using the $\chi^{2}$ test. Significance was set at $5 \%$.

\section{RESULTS}

We retrieved the provisional and definitive reports of 429 patients. Fifty eight intrauterine deaths, perinatal, and malformation necropsies were excluded from our study. Therefore, 371 necropsies were analysed, representing $61 \%$ of the "non-intrauterine deaths and perinatal/malformation necropsies" performed by the resident staff in that year. Of those, 214 were men and 157 were women; the mean age was 50.3 years, ranging from 1 to 92 . The mean number of diagnoses assigned for each necropsy was 10.7, ranging from four to 22 .

Table 1 shows the total number of diagnoses for each organ, the percentage of concordant and discordant/additional diagnoses, the percentage of diagnoses feasible only through histological analysis, and the inconclusive diagnoses after microscopic examination. The lung was the organ that received the highest number of diagnoses $(n=954)$ and the pancreas had the lowest number of diagnoses $(n=390)$. Interestingly, the lungs were not mentioned in only five of the necropsy reports.

As shown in table 1, the lung and the liver were the organs with the most frequent discrepancies between the gross and microscopic findings: $38.7 \%$ and $35.1 \%$, respectively. The lung showed a significantly increased frequency of discordance compared with all the other organs $(p \leqslant 0.009)$ except the liver $(p=0.19)$. Similarly, the frequency of discrepancies was higher in the liver when compared with all other organs $(\mathrm{p}=0.006)$, except the kidney $(\mathrm{p}=0.10)$ and lungs $(p=0.19)$. The brain was the organ with the lowest frequency of discrepancies when compared with all other organs $(\mathrm{p}=0.00)$.

In a small number of cases the diagnosis could only be achieved through microscopic analysis, with the highest frequency being found in the kidneys $(8.5 \%)$. The frequency of inconclusive gross and histological diagnoses was very low in all analysed organs.

We further classified the concordant cases into three categories (table 2$)$. The lung had the lowest $(0.9 \%)$ and the brain the highest $(48.1 \%)$ frequency of no macroscopic or histological alterations. The histological analysis contributed

Table 2 Concordant results in relation to the various organs

\begin{tabular}{|c|c|c|c|c|}
\hline Organ & Diagnosis* & $\begin{array}{l}\text { No gross or histological } \\
\text { abnormalities } \\
\mathrm{N}(\%)\end{array}$ & $\begin{array}{l}\text { Concordant diagnosis } \\
\mathrm{N}(\%)\end{array}$ & $\begin{array}{l}\text { Diagnostic refinement with histological } \\
\text { analysis } \\
\mathrm{N}(\%)\end{array}$ \\
\hline Lung & 564 & $5(0.9)$ & $473(83.9)$ & $86(15.2)$ \\
\hline Heart & 401 & $117(29.2)$ & $271(67.6)$ & $13(3.2)$ \\
\hline Brain & 364 & $175(48.1)$ & $165(45.3)$ & $24(6.6)$ \\
\hline Kidney & 365 & $64(17.6)$ & $258(70.9)$ & 42 (11.5) \\
\hline Liver & 341 & 52 (15.2) & $225(66)$ & $64(18.8)$ \\
\hline Spleen & 306 & 98 (32) & $169(55.2 \%)$ & 39 (12.8) \\
\hline Pancreas & 285 & $217(76.1)$ & $53(18.6 \%)$ & $15(5.3)$ \\
\hline
\end{tabular}


Table 3 Discordant results in relation to the various organs

\begin{tabular}{|c|c|c|c|c|}
\hline Organ & Diagnosis* & $\begin{array}{l}\text { Gross-histological } \\
\text { discrepant diagnoses } \\
\mathrm{N}(\%)\end{array}$ & $\begin{array}{l}\text { Not previously cited in the } \\
\text { macroscopic report } \\
\mathrm{N}(\%)\end{array}$ & $\begin{array}{l}\text { Macroscopic diagnosis not listed in the final } \\
\text { report } \\
\mathrm{N}(\%)\end{array}$ \\
\hline Lung & 369 & $62(16.8)$ & $242(65.6)$ & $65(17.6)$ \\
\hline Liver & 201 & 38 (18.9) & $132(65.7)$ & 31 (15.4) \\
\hline Kidney & 181 & 19 (10.5) & $127(70.2)$ & 35 (19.3) \\
\hline Heart & 140 & $12(8.6)$ & 100 (71.4) & $28(20)$ \\
\hline Spleen & 127 & $31(24.4)$ & $70(55.1)$ & $26(20.5)$ \\
\hline Pancreas & 97 & $10(10.3)$ & 71 (73.2) & $16(16.5)$ \\
\hline Brain & 61 & 10 (16.4) & $40(65.6)$ & 11 (18) \\
\hline
\end{tabular}

most to refining the final diagnosis in the lung and the liver, whereas it contributed the least in the heart.

Discordances were subdivided into three categories (table 3). Interestingly, most of the discrepancies were related to abnormalities not listed in the gross findings for all organs analysed. Moreover, a substantial proportion of the gross diagnoses were not listed in the final report.

There were concordance/diagnostic refinements between the gross and histological diagnoses of the major disease and the immediate cause of death in $89.5 \%$ and $76.3 \%$ of the cases, respectively. In $8.9 \%$ of the cases there were discordances related to the major disease and in $23.1 \%$ of the cases they were related to the immediate cause of death (table 4).

\section{DISCUSSION}

Few studies have addressed the issue of diagnostic changes after histological examination in different organs at necropsy. We found that histological analysis has a major impact on previously performed gross diagnosis. The frequency of gross versus histological discrepancies was $38.7 \%$ for the lungs, $35.1 \%$ for the liver, and $30.3 \%$ for the kidneys. In the liver and lung, histological analysis helped to refine the gross diagnosis in $18.8 \%$ and $15.2 \%$ of cases, respectively. For the kidneys, the final diagnosis could only be achieved through histological analysis in $8.5 \%$ of the diagnoses.

There is some discussion as to the necessity of histological sampling in routine necropsies. Histological sampling obviously increases $\operatorname{costs}^{10}$ and turnaround times, and consequently, some pathologists believe that histology may not always be necessary. ${ }^{6}$ In addition, in the UK, after the Alder Hey affair, there has been a decrease in the proportion of necropsies in which tissue is retained for histological analysis. ${ }^{16}$ However, recent guidelines on necropsy practice do recommend histological sampling of all major organs to confirm the macroscopic diagnosis, refine the cause of death, assist in clinical audit, and aid in the training of pathologists. ${ }^{17}$

We found that the lung was the organ where most diagnostic discrepancies or refinements occurred after microscopic examination. Indeed, pathologists who routinely perform necropsies will appreciate that the difficulties in rendering accurate diagnoses based on the macroscopic appearances of the lungs are not trivial. There are some entities that may be similar at gross examination, such as bronchopneumonia and diffuse alveolar damage. Other authors have previously reported some of the difficulties in gross examination of the lung, especially concerning the diagnosis of bronchopneumonia ${ }^{12}$ and acute pulmonary infections. ${ }^{13}$ Our results show a similar degree of discordance between gross and microscopic findings to that reported by Hunt et al when checking the diagnosis of bronchopneumonia-30.8\%. ${ }^{12}$ This is particularly important because the lungs are often pathologically altered at necropsy. In our study, the lungs were considered to be normal in only five of the total number of necropsies.

"We found that the lung was the organ where most diagnostic discrepancies or refinements occurred after microscopic examination"

We made similar observations in other organs, such as the liver and the kidney. Idalino et al, ${ }^{14}$ in a local study, compared macro and micro diagnoses in 100 necropsies and found that most of the discrepancies occurred in the same organs that we describe-the lung, kidney, and liver. It may be difficult to distinguish a benign from a malignant nodule in a cirrhotic liver by means of macroscopic examination only, and microscopy is essential to refine the diagnosis of the hepatopathies. Similarly, for the kidneys, microscopic examination is mandatory to diagnose and classify a glomerulopathy, for instance.

Analysis of the discrepant/additional diagnosis could provide information about necropsy quality in general. In a considerable proportion of cases in the discrepant category, diagnoses listed on the histological report were not described in the gross report; these included lung emphysema and myocardiosclerosis, lesions that in theory could have been seen macroscopically. It is possible that some of these differences could result from inaccuracy of the macroscopic examination and also inadequate gross necropsy reportingfor example, small subpleural emphysema in an elderly patient, which might not be thought worth mentioning. Alternatively, some of the diagnoses listed in the gross examination were not found in the histological analysis. This may be partially related to inadequate sampling, but also to inadequate reporting: a reference to a described macroscopic

Table 4 Impact of histological examination on macroscopically determined major disease and immediate cause of death in 371 necropsies

\begin{tabular}{lllll}
\hline & $\begin{array}{l}\text { Concordant diagnosis or with } \\
\text { diagnosis refinements } \\
\mathbf{N}(\%)\end{array}$ & $\begin{array}{l}\text { Discordant diagnosis } \\
\mathbf{N}(\%)\end{array}$ & $\begin{array}{l}\text { Histology needed } \\
\mathbf{N}(\%)\end{array}$ & $\begin{array}{l}\text { Remained inconclusive } \\
\mathbf{N}(\%)\end{array}$ \\
\hline Major disease & $332(89.5)$ & $33(8.9)$ & $3(0.8)$ & $3(0.8)$ \\
Immediate cause of death & $283(76.3)$ & $86(23.1)$ & $2(0.6)$ & $0(0)$ \\
\hline
\end{tabular}




\section{Take home messages}

- Histological analysis has a major impact on previously performed gross diagnosis at necropsy, especially in the lungs, liver, and kidneys

- Adequate sampling and histological analysis are important for necropsy quality

abnormality should always be found again in the final report. Possibly, some reluctance by the pathologists to alter a previous diagnosis may also play a role in this finding.

Our study has certain limitations. It was a retrospective analysis, looking at necropsy reports performed by different pathologists with different pathological backgrounds. There was broad but no systematic sampling, which will certainly have influenced our results. However, we believe that this situation is the closest to a routine necropsy teaching department. ${ }^{11}$ It is possible that necropsied cases were those with a high likelihood of benefiting from a microscopic analysis, leading to an overestimation in our results. However, this problem is inherent to all studies with a similar design and, we believe, it was minimised in our study by the high necropsy rate at our institution.

Our data show that microscopic analysis changed the reported cause of death in $23.1 \%$ of cases. Indeed, Veress et al reported that there is moderate agreement among pathologists regarding the immediate cause of death at gross necropsy, and suggested that macroscopic findings could be discussed among pathologists if the clinical picture cannot be explained satisfactorily, and that the clinicians should determine the cause of death after the necropsy. ${ }^{18}$

The heart, pancreas, and the brain were the organs with most frequent diagnostic agreement, and interestingly, with the least diagnostic refinements after histological examination. One possible explanation for these findings is that the heart and the brain are frequently affected by vascular disorders, leading to acute or chronic ischaemic or haemorrhagic changes that are usually obvious macroscopically. Similarly, pancreatitis or tumour masses, probably the most common diseases of the pancreas, are not difficult to diagnose macroscopically.

In summary, we have shown that histological analysis has a major impact on macroscopic diagnosis, altering and refining previous diagnoses, especially in the lungs, liver, and kidneys. Histological analysis also results in changes to the immediate cause of death and underlying diseases in a considerable proportion of cases. Epidemiological data, hospital quality control programmes, and judgment of malpractice may be based upon necropsy reports. Moreover, in teaching hospitals necropsies have an important didactical role. Adequate necropsy performance, sampling, and reporting are essential to make necropsy the real final audit.

\section{ACKNOWLEDGEMENTS}

Supported by CNPq and LIM05-HCFMUSP.

\section{Authors' affiliations}

F D C Bernardi, P H N Saldiva, T Mauad, Department of Pathology, Sao Paulo University Medical School, Sao Paulo SP, $01246-903$ Brazil

\section{REFERENCES}

1 Burton JL. The autopsy in modern undergraduate medical education: a qualitative study of uses and curriculum considerations. Med Educ 2003;37:1073-81.

2 Goldman L, Sayson R, Robbins S, et al. The value of autopsy in three medical eras. N Engl J Med 1983;308:1000-5.

3 Anderson RE, Fox RE, Hill RB. Medical uncertainty and the autopsy: occult benefits for students. Hum Pathol 1990;21:128-35.

4 Modelmog D, Rahlenbeck S, Trichopoulos D. Accuracy of death certificates: a population-based, complete-coverage, one-year autopsy study in East Germany. Cancer Causes Control 1992;3:541-6.

5 Zarbo RJ, Baker PB, Howanitz PJ. The autopsy as a performance measurement tool-diagnostic discrepancies and unresolved clinical questions. A CAP Qprobes study of 2479 autopsies from 248 institutions. Arch Pathol Lab Med 1999; 123:191-8.

6 Schned AR, Mogielnicki RP, Satuffer ME. A comprehensive quality assessment program on the autopsy service. Am J Clin Pathol 1986;86:133-8.

7 Vujanic GM, Cartlidge PH, Stewart JH. Improving the quality of perinatal and infant necropsy examinations: follow up study. J Clin Pathol 1998;51:850-3.

8 Zarbo RJ, Baker PB, Howanitz PJ. Quality assurance of autopsy permit form information, timeliness of performance, and issuance of preliminary report. $A$ College of American Pathologists Q-Probes study of 5434 autopsies from 452 institutions. Arch Pathol Lab Med 1996;120:346-52.

9 Shojania K, Burton E, McDonald K, et al. The autopsy as an outcome and performance measure, Evidence Report/Technology Assessment No.58 (prepared by the University of California at San Francisco-Stanford evidencebased practice center under contract no.290-97-0013).AHRQ Publication No.03-E002. Rockville, MD: Agency for Healthcare Research and Quality, October, 2002.

10 Reid WA. Cost effectiveness of routine postmortem histology. J Clin Pathol 1987:40:459-61.

11 Burton JL, Underwood JCE. Necropsy practice after the "organ retention scandal": requests, performance, and tissue retention. J Clin Pathol 2003;56:537-41

12 Hunt CR, Benbow EW, Knox WF, et al. Can histopathologists diagnose bronchopneumonia? J Clin Pathol 1995;48:120-3.

13 Hjorth L, Jensen HS, Noes H. Acute pulmonary infections at autopsy. A study of clinical and macroscopic diagnoses at autopsy compared with microscopic autopsy findings. Ugeskr Laeger 1995;157:6873-6.

14 Idalino CV, Gomes EE, Cury PM. Estudo comparativo dos diagnósticos macro e microscópicos e possíveis causas de erro. (Comparative study of gross and microscopic diagnosis of autopsies: analysis of difficulties and eventual error.) Jornal Brasileiro de Patologia e Medicina Laboratorial 2004;40:325-31.

15 Xavier AC, Siqueira SA, Costa $\amalg$, et al. Missed diagnosis in hematological patients - an autopsy study. Virchows Arch 2005;446:225-31.

16 Burton JL. The Alder Hey affair: implications for pathology practice. J Clin Pathol 2001;54:820-3.

17 Guidelines on autopsy practice. Report of a working group of the Royal College of Pathologists, September 2002 (available at: http:// www.rcpath.org/index.asp?PagelD = 240)

18 Veress B, Gadaleanu V, Nennesmo I, et al. The reliability of autopsy diagnostics: inter-observer variation between pathologists, a preliminary report. Qual Assur Health Care 1993;5:333-7. 